\title{
Cardiology Tele-Homecare Monitoring In Older Patients with Congestive Heart Failure Enhances the Quality of Care in Chronic Disease Management- Pilot Project
}

\author{
Kaladjudjevic $\mathbf{M}^{1}$, Malatesta $\mathrm{G}^{2 *}$, Rossi $\mathrm{L}^{3}$ and Antonicelli $\mathrm{R}^{2}$ \\ ${ }^{1}$ Facoltà di Farmacia, Centro di Telemedicina e Telefarmacia, Università di Camerino, Italy \\ ${ }^{2}$ U.O. di Cardiologia, Centro di Telemedicina, Istituto Scientifico INRCA, Ancona, Italy \\ ${ }^{3}$ Lab. di Bioingegneria, Bioinformatica e Domotica Istituto Scientifico INRCA, Ancona, Italy
}

Received: January 13, 2015; Accepted: August 16, 2015; Published: August 26, 2015

*Corresponding author: Dr. Gelsomina Malatesta, University Cardiology Unit, Centro di Telemedicina, Istituto Scientifico INRCA, INRCA, Ancona, Italy. Tel: + 3386362648; E-mail: G.MALATESTA@inrca.it

\begin{abstract}
Background: Several chronic diseases are highly associated with disability, requiring a high level of health-care service. Congestive Heart Failure (CHF) represents one of the most frequent chronic diseases associated with poor quality of life, reduced social activity and patient mobility, especially in old patients. During CHF management, both the patient and caregivers should hold an active role in the type of necessary care need. Considering that the majority of patients (or caregivers) are not completely aware of the necessary care plan, direct medical supervision is crucial. According to the recent national guidelines on tele-homecare, an in-depth reorganization of health care is necessary to identify needs from the hospital setting to homecare management in urban area, which in turn will play an important role on improving personal independency levels and quality of life.
\end{abstract}

Aim of our study: In contemporary literature there is a lack of clinical trials for evaluating the impact of telemedicine on quality of life in elderly population affected by cardiovascular disease. The aim is to identify variables associated with improved quality of life of older people with CHF who lives in urban area of medium sized city, (Ancona, Italy).

Methods: Tele-homecare for CHF combined with health education courses, addressed to both the patient and caregiver, followed by weekly tele-monitoring of patient vitals. A web-based patient interface technology using medical devices equipped with a software platform, capable of monitoring, recording and sending to cardiology department. The study started in June 2014 and lasted for 12 months.

Results: This is a pilot study with patients undergoing training sessions to recognize acute signs and symptoms of heart failure including feedback on learning and self-management skills of the disease. By using Kansas city Cardiomyopathy questionnaire before and after intervention, we obtain improvement in symptom frequency, stability and symptom burden score, as well as in self efficacy and quality of life score.

Keywords: Telemedicine; Monitoring; Congestive Heart Failure; Elderly People; Kansas City; Cardiomyopathy; Questionnaire

\section{Introduction}

Chronic disease treatment plans aims to reach the following goals:

i) Preventing disease progression,

ii) Prolonging life, and

iii) Improving patient quality of life.

The quality of life of elderly population, who are more affected by chronic diseases and cardiovascular disease, is not satisfying. In future the large group of rapidly growing elders will be associated with an increased risk of disability levels mainly caused by the major chronic disease. Cardiovascular disease is considered a major cause of disability predicting 18 (106) DALY (disability adjusted life years) by the year 2020 according to Global Burden of Disease Study [1]. Chronic heart failure is among most important syndromes of aging and is associated with limited mobility, frequent re-hospitalizations and higher mortality rates after hospital discharge. In following years, this aging population will increase healthcare and social costs, with a parallel rise in the prevalence of $\mathrm{CHF}$ and related disabilities. It is commonly observed that the number of chronic co morbidities in older persons cause the need for continuous care measures by caregivers. A centered patient care approach allows for a significantly effective decision-making process that promotes an active involvement of both patients and their caregivers. The health education intervention provided through the centered patient care, has demonstrated to provide improved patient compliance to the chronic therapy measures [2]. Effectiveness of healthcare intervention depends on active patient participation toward understanding the disease complexity.

Remote monitoring through tele-homecare [3]is a promising intervention, which theoretically should improve quality of life of elderly patient, while promoting real time clinical monitoring of patient's vital signs. 
We evaluated the state of art of research on quality of life of elderly affected with chronic heart failure, using telecardiology interventions, then we conducted a pilot project and evaluated quality of life modification before and after the pilot trial, using Kansas City Cardiomyopathy Questionnaire [4].

\section{Methodology}

We conducted systemic literature review. Systematic searches of the published articles were done using the PubMed,MEDLINE, CINAHL, SCI, and Cochrane Library electronic databases. We estimated the volume of research published in the area of quality of life evaluation with telecardiology intervention, by using different keywords (telemedicine; telecardiology; telecardiology and quality of life, quality of life and Chronic Heart Failure). Using only a word "Telecardiology" within abstract, title and keywords, Cochrane library showed only 1 results among 34181 abstracts. With the words "Telecadiology and Quality of life", Cochrane library showed 1 results among 843852 abstracts (Table 1).

\section{Aim of the study}

The purpose of the study is to evaluate potential improvement of quality of life in CHF patients using tele-homecare technologies who resides in urban area, as well as, to improve patients/ caregivers self efficacy at home in order to manage better her/ his disease.

\section{Pilot Project}

Patients who were admitted in the acute care were included in the study from the Istituto Nazionale di Riposo e Cura per Anziani (INRCA) Cardiology division in Ancona, Italy. This unit has over 500 admissions yearly, with primary diagnosis of CHF, and are mostly found in the very old population (mean age 84 years). The Cardiology division provides a Telecardiology service since 1999. There is a significant raise in healthcare cost, in the number of frequent re-hospitalizations in this population due to patient post discharge vulnerability which is up to $22 \%$ within first month following discharge. Such a large percentage of re-hospitalization has lead to the increased use of telemedicine monitoring following an acute care discharge in these older patients. At the same time, this type of technology allows to perform the testing of important clinical trials which are hard in a rapidly growing population.

The Cardiology division at INRCA in Ancona, Italy sponsors this study. Physician- patient interviews were conducted in the presence of caregiver to enroll in study protocol. The study protocol has been organized by a multidisciplinary group of trained staff of cardiologists, medical engineers and nurses. The clinical trial protocol has been submitted to Ethical committee.

\section{Study population}

The study consists of patients with a diagnosis of CHF and frequent re-hospitalizations (at least three re-hospitalization in last year), over the age of 80 years, living at home, in a selfsufficient manner or assisted by a caregiver or family member. The study includes data from the hospital stay in patients and data from the telemedicine kit. Inclusion criteria were as follows: at least 3 re-hospitalization with primary diagnosis of heart failure within last year, $\geq 80$ years of age; II-III class NYHA; HF of different etiology (ischemic, valvular, hypertensive); presence of one or more of the following co-morbidities: diabetes, renal insufficiency, hypertension, anemia; assisted by caregiver, (formal or informal) who have basic level of information technology use; internet access at home.

Participants were excluded according to the following exclusion criteria: age $<80$ years; recent acute myocardial infarction; severe dementia; severe renal insufficiency (glomerular filtration < 20); chronic dialysis; cancer with short life expectancy ( $<1$ year); absence of a caregiver (formal or informal). After screening period conducted during patient's hospital stay, every patient and caregiver have to sign inform consent before enrolling in the study.

\section{Endpoints}

Primary Objectives: To evaluate Quality of life using Kansas City Cardiomyopathy questionnaire before and after telehomecare intervention.

Intervention: In order to participate in the study, every caregiver and patient was required to undergo the following training modules: how to use telemedicine devices, recognizing heart failure symptoms and management. A structured brochure containing all major information regarding $\mathrm{CHF}$, how to place ECG leads and electronic stethoscope was given to every patient and caregiver. Detailed modules are presented in table 2. Every patient has received a list with telephone and email contacts of responsible person who should be contacted in case of technical or other problems.

Table 1: Results from Large database search.

\begin{tabular}{|c|c|c|c|c|c|}
\hline words & PUBMED & COHRANE & CINAHL & SCI & MEDLINE \\
\hline Telecardiology & 169 & 1 & 14 & 190 & 137 \\
\hline Telemedicine & 18363 & 9 & 0 & $\mathrm{~N} / \mathrm{A}$ & $\mathrm{N} / \mathrm{A}$ \\
\hline Telecardiology +QOL & 13 & 1 & 0 & 15 & 519 \\
\hline Telecardiology +QOL+CHF & 8 & 0 & 3 & 1 & 9 \\
\hline
\end{tabular}

The database research showed a scarcity of clinical trials that evaluate quality of life of elderly affected by CHF and use of tele- home care intervention. Despite of the scarcity of trials we found, a large numbers of clinical studies have shown that telemedicine may offer a real time titration and better medication compliance. We conducted a pilot trial that enrolled elderly population affected by CHF with aim to evaluate quality of life changes before and after the telemedicine intervention. 
During Telemedicine kit training program, each patient was required to complete two questionnaires. The first questionnaire from Kansas City Cardiomyopathy, for patient self-assessment measurement, evaluates quality of life improvement in CHF patient before and on the end of a pilot project. The surveys were performed during the first visit, and at the end of study period.

A telemedicine platform supporting multispecialty teleconsultations and tele-home care was used. The software was developed and to be used by people who are without medical skills, like patients and caregivers. The technical system platform has to be configured at the moment of deployment to the user. The user interface has been developed to take into account the simplicity of using the mandatory characteristics. The software allows in real time the storage and sending data without data loss, also in the case of lack of connectivity. It integrates different types of medical devices tailored for the patient. Every kit contains medical device (blood pressure device, oxygen saturation device, weight control device, ECG-12 leads, electronic stethoscope) (Figure 1) and tablet with software.

The Telemedicine kit is very easy to use. After an internet connection, the software application opens instantly and displays 4 images representing access to separate pages of 4 vital signs measurement (saturation, blood pressure, weight and ECG). The patient or caregiver, once a week, was required to perform vital sign measurements. All data measured with medical devices were transmitted to a software program installed on tablet using a Bluetooth connection. Data transmission from medical devices to software appeared in defined boxes on display. Patients may control the data transmitted on tablet to control that the medical device measured the corresponding data. This procedure was repeated for 4 measurements. Data was then transmitted directly to the cardiology division and every patient (user) received a confirmation message that measurement had been sent successfully (Figure 2).

For ECG measurements, the Cardio line Microtel was utilized and has shown to be highly predictable of any pathological changes. As previously mentioned, the brochure contains images on how to place ECG leads. In the case erroneous positioning of ECG leads, caregivers were contacted by the cardiology division in order to repeat ECG to achieve a correct reading.

On the other hand, both caregivers and patients were instructed to directly contact a cardiologist via teleconsultation option, if there is a presence of worsening of heart failure symptoms such as, dyspnea, decrease in diuresis, ankles edema, rapid or irregular heartbeat or reduced ability to exercise. Teleconsultation permits video-teleconference and remote auscultation with electronic stethoscope that allows to record pulmonary activity. Caregiver participation is essential for teleconsultation as he/she can give more detailed patient information and is trained to use electronic stethoscope to perform recording and transmission of lung sound. The detailed brochure also contain images and descriptive explanation on how to use the electronic stethoscope.

Every physician was allowed to access the data on a daily basis in order to control the list of patients enrolled in the trial. Using a secure protected system, all study physicians could also consult transmitted data of each patient regarding vitals, as well as visualize all data over time graphically using a Telemedicine Platform. If required, medical doctor are contacted by the patient to confirm, treatment optimization, need for hospitalization or medical examination.

\section{Statistical analysis}

This was an observational study which lasted 12 months. Study intervention included: a health education course (1st day) and weekly telemonitoring of vital signs starting from 3rd day. In order to estimate the effect of intervention on target groups, without random assignment, in an observational study, we used a quasi-experimental design. The problem of any quasi-experimental design is a lack of internal validity. In order to reduce number of confounding factors, a propensity score matching technique was used. Patients with the same inclusion and exclusion criteria were selected from Cardiology division database to be compared with patients in intervention group at the end of the trial.

\section{Results}

The protocol began in June 2014; the period of enrollment

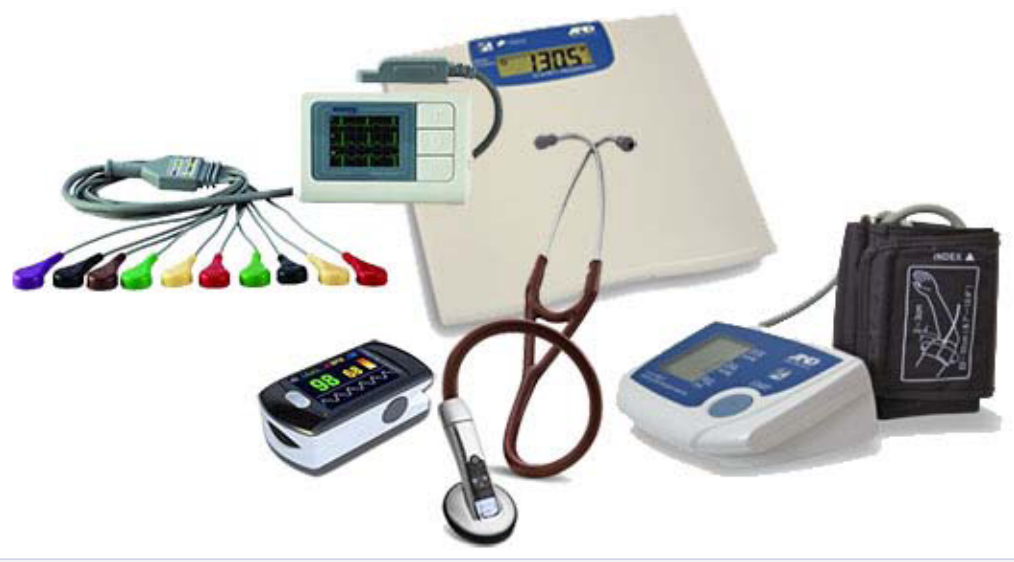

Figure 1: Telemedicine kit for the patients. 


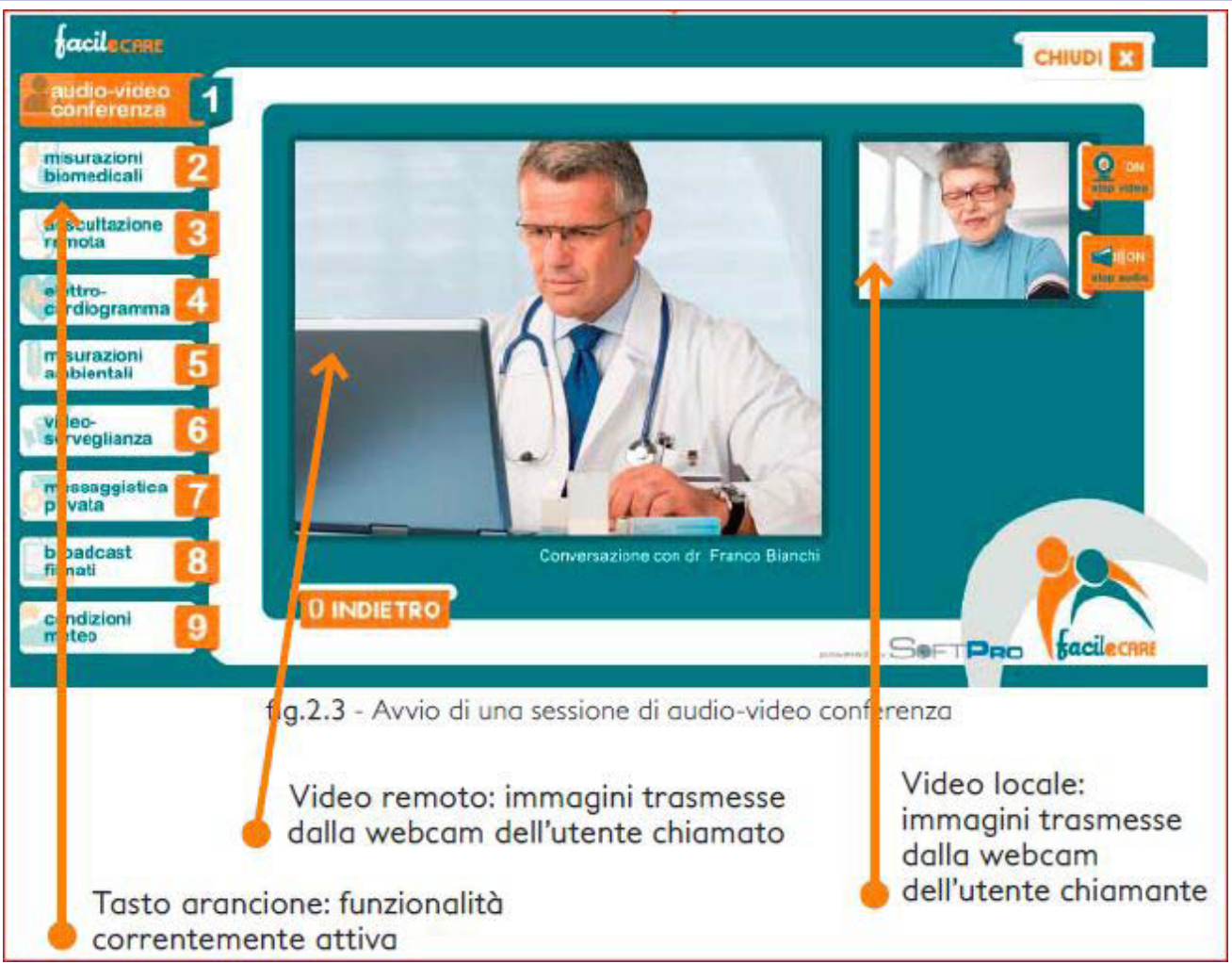

Figure 2: Remote video with images transmitted by the called party feature, active current image transmitted by the user caller.

Table 2: Structured training modules for study protocol.

\begin{tabular}{|c|c|}
\hline Course & MODULES \\
\hline \multirow{5}{*}{ Basic elements } & Elements of psychology and communication \\
\hline & Safety of domestic environment \\
\hline & $\begin{array}{l}\text { How to take care of elderly person with specific } \\
\text { needs }\end{array}$ \\
\hline & Proper nutrition of the elderly \\
\hline & How to obtain services and assistance \\
\hline \multirow{2}{*}{ Heart Failure } & Heart failure basic knowledge \\
\hline & Living with heart failure \\
\hline \multirow{5}{*}{ Advanced } & Problems solving and negotiation \\
\hline & Elements of first aid \\
\hline & Advanced skills on management of elderly persons \\
\hline & Information technology \\
\hline & Use of telemedicine system \\
\hline \multirow{2}{*}{ Life style } & Aging and promotion of healthy Life style \\
\hline & Healthy Life style for elderly with specific pathology \\
\hline
\end{tabular}

has been concluded by September 2014. Total number of patient enrolled was 10 , but 2 out of 10 died before using the Telehomecare system, thus 8 patients have been monitored during 12 months of pilot project ( June 2014 - June 2015) and have sent over 750 measurements of vital signs, 170 ECG telemonitoring and 150 teleconsultations. During pilot project, the initial perception of Tele-homecare demonstration was positive, family members were interested in learning how to measure, send measurements and to be more involved in disease management (Table 3).
The Kansas City Cardiomyoptahy Questionnaire (KCCQ) (Table 4) has a high internal consistency (Cronbach score 0,90) and has showed to be more sensitive to the clinical changes, particularly in patients post discharge [5]. The KCCQ is a disease specific health status instrument for patient affected by heart failure, represented by 23 questions which measure symptoms (frequency, severity and recent change over time), physical limitations, social functioning and patient's sense of self efficacy and quality of life Daniela Miani et al. [4] Confrontation of

Table 3: Patent Characteristics for tele-home care demonstration.

\begin{tabular}{|c|c|c|}
\hline \multicolumn{2}{|c|}{ Patent Characteristics } & \multirow[t]{2}{*}{$n=8$} \\
\hline Age & $85(80-94)$ & \\
\hline \multirow{2}{*}{ Gender } & Male & $30 \%$ \\
\hline & Female & $70 \%$ \\
\hline \multirow{3}{*}{ Nyha Class } & I class & \multirow{3}{*}{$100 \%$} \\
\hline & II class & \\
\hline & III class & \\
\hline Ejection Fraction & $45.00 \%(30-50 \%)$ & \\
\hline \multirow{3}{*}{ Etiology } & Ischemic & $80 \%$ \\
\hline & Valvular & $50 \%$ \\
\hline & Dilated & $50 \%$ \\
\hline $\begin{array}{l}\text { Previous } \\
\text { Hospitalization }\end{array}$ & $\begin{array}{l}\text { At least } 3 \\
\text { hospitalization within } \\
\text { last year, with primary } \\
\text { diagnosis of CHF }\end{array}$ & \\
\hline \multirow{2}{*}{ Therapy } & ACE inhibitor & $90 \%$ \\
\hline & Beta-Blockers & $90 \%$ \\
\hline
\end{tabular}


symptoms stability and symptoms frequency score before and after has showed a slight improvement (between 1- 2 points). The self efficacy score were measured according to the guidelines of KCCQ Daniela Miani et al. [4] by summarazing scores on questions 15 and 16 before and after pilot project. The Self efficacy score were improved after pilot project, demonstrating that health education courses have improved knowledge about disease and its management, while telemonitoring [6] improved the accessibility to secondary care - cardiology department through video teleconsultation.

Example: Patient 01, Question 15 ( Kansas City Cardiomyopathy Questionaires)

Heart Failure Symptoms can worsen for a number of reasons. How sure you are that you know what to do, or whom to call, if your heart failure gets worse?

Answer before pilot project: Not at all sure (score 0)

Answer after pilot project: Completely sure (score 4)

Patient 01, Question 16 (Kansas City Cardiomyopathy Questionaires)

How well do you understand what things you are able to do to keep your heart failure symptoms from getting worse? $0)$

Answer before pilot project: do not understand at all (score

\section{Answer after pilot project: Mostly understand (score 3)}

The heath education course improved awareness of important chronic heart failure symptoms; this has been observed by increased score for question 15 and 16 after 12 months of observation. Initially the answers on self efficacy questions were demonstrating limited knowledge about symptoms and how to manage it, as most of the patients have answered that they are not sure what they should do. After 12 months of monitoring and teleconsultation which improved accessibility to secondary healthcare service, patients have been empowered to manage heart failure more effectively, that has been demonstrated by their answers where they declare they are completely sure how to manage heart failure.

The further steps planned, after pilot project, is to organize

Table 4: Kansas City Cardiomyopathy Questionnaire.

\begin{tabular}{|c|c|c|}
\hline \multirow{2}{*}{$\begin{array}{l}\text { Kansas City Cardiomyopathy } \\
\text { Questionnaire }\end{array}$} & \multicolumn{2}{|c|}{ n=8 } \\
\hline & Before & After \\
\hline Symptoms Stability score & $55(20-100)$ & $62,5(20-100)$ \\
\hline Symptoms frequency score & $36,3(0-100)$ & $47,5(0-100)$ \\
\hline Symptoms burden score & $43,3(0-100)$ & $53,3(0-100)$ \\
\hline Quality of life score & $28,3(0-80)$ & $37(0-80)$ \\
\hline Social Limitation domain & $10(0-80)$ & $21(0-80)$ \\
\hline Self efficacy domain & $34(0-80)$ & $51(0-100)$ \\
\hline Combined symtoms score & $45(0-100)$ & $55(0-100)$ \\
\hline Overall summary domain & $34,5(0-100)$ & $45(0-100)$ \\
\hline
\end{tabular}

focus group with patients and their caregivers with an aim to acquire more information through semi structure interviews about their problems during a pilot project. The semi structured interview will give more in depth information on how to improve protocol and what patients with heart failure require in order to be more empowered and involved in management of heart failure.

\section{Discussion}

Frequent hospitalizations related to heart failure have significant impacts on quality of life and clinical prognosis. The disease management of patient with CHF requires tight monitoring and optimization of therapy that need to include diverse professionals, especially cardiologists and GPs. The diversity of many studies $[1,2,7,8,9,10]$ carried out to optimize the management of outpatient or home care of patients with heart failure are related to the type of post-hospital discharge. Programs generally include periodic phone calls from nurses and regular visits to adjust the patient or domiciliary care in order to improve patient motivation to reach optimal self-management. However, elderly CHF patients [11], need constant surveillance in order to identify those at risk of hospitalization [12] or rapid functional decline because of such significant higher risk of exacerbation of symptoms and increased mortality. Caregivers are usually family members, not medically educated person, who does not have adequate information and knowledge about disease, related risk factors, symptoms and appropriate management necessary to prevent worsening of heart failure. We have analyzed and evaluated potential interventions that may interfere to resolve these problems such as combining health education through secondary care (cardiology and geriatric care) and clinical monitoring of patients with CHF [13]. As a result in our pilot project the major improvement has been demonstrated in self efficacy domain, the reason for it, as patients have explained was the $1^{\text {st }}$ day education course that gives them more knowledge about disease and how to manage it. Our objective is to improve patient understanding to maintain a more active and independent role in the management of CHF [14]. This study highlights that health education and health promotion courses may enhance self-management in patient's behavior, while telemonitoring may allow correct medical interventions in timely manner with potential to enhance of quality of life.

The pilot project has its limitations: the small number of patients, that constrained any possibility to apply statistical analysis, a majority of patients were affected by NYHA III, this level of CHF syndrome doesn't give a lot of opportunity for physical improvement especially in population over 85 years of age, who are affected by other co morbidities too. Despite limitations we believe that larger sample of patients, with NYHA class II or I, and who are affected by only one co morbidities, could give more significant results [15].

\section{Conclusion}

Telemedicine allows to use for continuous healthcare related diseases, especially for elders with CHF. A large clinical trials using telemedicine that evaluates quality of life will be necessary 
for monitoring and consulting elderly populations. At the moment, INRCA is aiming to provide such answers. This protocol underlines that secondary prevention intervention combined with health education may provide better patient and caregiver empowerment of healthcare and improvement of quality of life parameters by utilizing the advantages of new technologies in older patients.

\section{References}

1. Murray CJ, Lopez AD. Alternative projections of mortality and disability by cause 1990-2020: Global Burden of Disease Study. Lancet. 1997; 349(9064): 1498-504.

2. Morisky DE, Green LW, Levine DM. Concurrent and predictive validity of a self-reported measure of medication adherence. Med Care. 1986; 24(1): 67-74.

3. TELEMEDICINA Linee di indirizzo nazionali. Ministero della Salute 2014

4. Miani D, Rozbowsky P, Gregori D, Pilotto L, Albanese MC, Fresco C, et al. The Kansas City Cardiomyopathy Questionnaire : Italian Translation and Validation. Ital Heart J; 4(9): 620-6.

5. Fried LP. Epidemiology of aging. Epidemiol Rev. 2000; 22(1): 95-106.

6. Cleland JG, Louis AA, Rigby AS, Janssens U, Balk AH; TEN-HMS Investigators. Noninvasive Home Telemonitoring for Patients with Heart Failure at High Risk of Recurrent Admission and Death The Trans-European Network-Home-Care Management System (TENHMS) Study. J Am Coll Cardiol. 2005; 45(10): 1654-64

7. Anderson C, Deepak BV, Amoateng-Adjepong Y, Zarich S. Benefits of comprehensive inpatient education and discharge planning combined with outpatient support in elderly patients with congestive heart failure. Congest Heart Fail. 2005;11(6): 315-21.

8. Phillips CO, Wright SM, Kern DE, Singa RM, Shepperd S, Rubin HR
Comprehensive discharge planning with postdischarge support for older patients with congestive heart failure: a meta-analysis. JAMA. 2004; 291(11): 1358-67.

9. Kornowski R, Zeeli D, Averbuch M, Finkelstein A, Schwartz D, Moshkovitz $\mathrm{M}$, et al. Intensive home-care surveillance prevents hospitalization and improves morbidity rates among elderly patients with severe congestive heart failure. Am Heart J. 1995; 129(4): 762-6.

10. Antonicelli R, Testarmata P, Spazzafumo L, Gagliardi C, Bilo G, Valentini $\mathrm{M}$, et al. Impact of telemonitoring at home on the management of elderly patients with congestive heart failure. J Telemed Telecare. 2008; 14(6): 300-5. doi: 10.1258/jtt.2008.071213.

11. Rector TS. Patients' self-assessment of their congestive heart failure. Part 2: Content, reliability and validity of a new measure, the Minnesota Living with Heart Failure questionnaire. Heart Failure. 1987;198-209.

12. Dendale P, De Keulenaer G, Troisfontaines P, Weytjens C, Mullens W, Elegeert I, et al. Effect of a telemonitoring-facilitated collaboration between general practitioner and heart failure clinic on mortality and rehospitalization rates in severe heart failure: the TEMA-HF 1 (TElemonitoring in the MAnagement of Heart Failure) study. Eur J Heart Fail. 2012; 14(3): 333-40. doi: 10.1093/eurjhf/hfr144.

13. Clark RA, Inglis SC, McAlister FA, Cleland JG, Stewart S. Telemonitoring or structured telephone support programmes for patients with chronic heart failure: systematic review and meta-analysis. BMJ. 2007; 334(7600): 942.

14. Shah NB, Der E, Ruggerio C, Heidenreich PA, Massie BM. Prevention of hospitalizations for heart failure with an interactive home monitoring program. Am Heart J. 1998; 135(3):373-8.

15. Di Lenarda A, Scherillo M, Maggioni AP, Acquarone N, Ambrosio GB, Annicchiarico $\mathrm{M}$, et al. Current presentation and management of heart failure in cardiology and internal medicine hospital units: A tale of two worlds-The TEMISTOCLE study. Am Heart J. 2003; 146(4): E12. 